\title{
A Look Back at the First Year of the New Maintenance of Certification Program for Family Physicians
}

\author{
Jane Ireland
}

Since the inception of the American Board of Medical Specialties (ABMS) almost 100 years ago, each of its member boards has either explicitly or implicitly addressed the issue of enhancing and assuring physician quality by setting standards for training and cognitive knowledge for their specialties. However, a number of reports issued in the late 1990s once again raised concerns about the health care system in the United States and cited a need for change to improve the delivery of health care to Americans. In response to these concerns, the American Board of Medical Specialties adopted a requirement that each of its 24 member boards develop specific mechanisms for implementing a Maintenance of Certification (MOC) process. As a result, the American Board of Family Medicine (ABFM), formerly the American Board of Family Practice, developed appropriate tools to assess and increase the depth and breadth of knowledge that family physicians use in practice on a daily basis, as well as to focus their knowledge base in key areas.

As we move into an era when even greater national emphasis is being placed on the demonstration of quality, the ABFM believes that Maintenance of Certification for Family Physicians (MCFP) will help board-certified family physicians provide even better care to their patients by continuously measuring the 6 general competencies as acknowledged by the Accreditation Council for Graduate Medical Education and the ABMS: patient care, medical knowledge, interpersonal and patient communication, professionalism, practicebased learning and improvement, and systemsbased practice. Each of the components of the MC-FP has been created to assure the public that physicians who hold our certificates continue to meet the highest standards and that their training, experience, and knowledge are sufficient to deliver care of the highest quality to people and their families.

\begin{abstract}
"The ABMS continues to work on behalf of its ambitious agenda to improve physician quality through its maintenance of certification program. Reasonable empirical evidence shows that certification and maintenance of certification programs will improve quality.... our polling data suggest the public is convinced that there is a connection, no doubt swayed by common sense."
\end{abstract}

ҰAMA 2004;292:9

\section{Implementation of MC-FP}

When the ABFM Board of Directors began planning for the implementation of these requirements, they were very cognizant of the need to avoid imposing unnecessarily burdensome requirements on Diplomates. MC-FP has been designed to take no more time than the recertification process that has been in place for more than 30 years; in many respects, it is very similar to the old process. However, various elements have been improved and updated.

To ease the transition into this new process, the Board of Directors decided that entry of current Diplomates into the MC-FP process would be staggered over 7 years. A new group will start the process each year, beginning the year after they recertify. Diplomates who recertified in 2003 entered MC-FP January 1, 2004, along with those who were certified for the first time in 2003. Those who were certified or recertified in 2004 entered the process in January 2005. This schedule will continue until all Diplomates are participating by January 2010.

\section{Differences between MC-FP and the Prior Recertification Process}

The major difference between MC-FP and the prior recertification process is that Diplomates are 
asked to participate in selected components on a regular basis (namely, the Self-Assessment Modules [SAMs] in Part II). Most of the other components necessary for the ABFM to meet the MOC requirements created by ABMS were already in place in some form.

The first requirement of MC-FP is proof of professional standing. The ABFM has always required a full, unrestricted license to maintain certification, and this satisfies the ABMS requirement.

The ABMS also requires a process to ensure that the ABFM Diplomates' knowledge and skills are updated on a more ongoing basis than has been done in the past with the 7-year examination cycle. To accomplish this requirement, the ABFM has created new assessment tools that incorporate active learning, feedback, and application, and these new tools are now available on the Internet. Six of these Self-Assessment Modules, or SAMs, must be completed during the 7-year MC-FP cycle, and the requirements have been designed to encourage Diplomates to complete one SAM per year rather than doing several near the end of the MC-FP cycle. It is important to note that Diplomates will receive $\mathrm{CME}$ credit for completing these modules and the Performance in Practice modules in Part IV. These credits can be applied against the 300hour CME requirement for the 7-year MC-FP cycle, which remains unchanged. Because these modules can be completed from the convenience of the physician's home or office computer, they will offset some of the CME cost that would have been required to meet the 300-hour CME requirement. Diplomates can currently select from a menu of 4 available SAMs: Asthma, Coronary Artery Disease, Diabetes, and Hypertension.

The third ABMS requirement is that physicians complete an objective assessment of knowledge in their field on a periodic basis, and the ABFM has required this of its Diplomates from the beginning. Family physicians have been required to pass a written examination every 7 years to maintain their certification.

The final requirement is assessment of performance in practice. In some ways, this requirement is similar to the Computerized Office Record Review (CORR) process that has been required for recertification by the ABFM. This process has been improved significantly with the development of the Performance in Practice Modules (PPMs) that will replace CORR, and we believe that Diplomates will find it more useful and more applicable to practice. The Performance in Practice modules will be required once every 7 years, and the first 2 modules, Diabetes and Hypertension, are currently available. Physicians who are not responsible for providing continuity of care to patients will fulfill this requirement by completing an alternative activity the ABFM will develop for this purpose. This group includes those in administrative positions, physicians working in emergency departments or urgent care centers, and others.

\section{Improvements in the SAMS}

Since their online debut in January 2004, the Self Assessment Modules (SAMs) have seen more than 20 modifications. Using feedback from its Diplomates, the ABFM continues to improve the SAM application. Listed below are the changes that have been made to both the knowledge assessment and the clinical simulation portions of the SAMs.

\section{Knowledge Assessment}

- Obtained permissions to use the reference articles without log on and/or payments.

- Converted the references to a PDF file to allow physicians to download the references for off-line review.

- Updated the tutorials to provide more comprehensive presentations of the knowledge assessment and clinical simulation components.

- Developed a "Road Map" to provide physicians with a strategy for navigating within the SAMs process.

- Provided the capability of allowing physicians to review the study guides while taking the knowledge assessment module.

- Developed a critique for each of the items that provides physicians with a concise, comprehensive review of the materials required to correctly respond to the questions in the SAMs as well as links to the references from which the question originated.

- Eliminated the requirement that physicians view the references before seeing the critiques.

- Developed the capability of allowing physicians to preview the first 15 questions in a module and then change to another module without an additional module fee.

- Developed the capability of allowing physicians to review all the items with critiques after successfully completing a module. 
- Developed the capability of allowing physicians to download all the items and references links in a PDF file so that they can take the module offline and then enter their answers online.

- Developed the capability of presenting a progress screen that allows physicians to branch directly to the items that they answered incorrectly.

- Reduced the local system requirements used for graphics presentation.

- Implemented significant changes in the software to make it more user-friendly for Apple Macintosh users.

- Expanded the number of web browsers that are supported in the presentation of the SAMs.

- Added functionality to provide physicians with the opportunity not only to receive CME credit for the SAMs but also to have the ABFM automatically notify the American Academy of Family Physicians (AAFP) of the CME hours (this functionality includes the opportunity to evaluate the SAMs online).

- Developed a hot-support capability that assists the Help Desk in diagnosing and solving physician interface problems.

\section{Clinical Simulation}

\section{Short-Term}

- Eliminated heart, lung, and bowel sound Java applets included in the initial interface because of problems with security settings in users' local networks.

- Updated synonyms used to match various terms that Diplomates use to express queries and order interventions.

- Added additional diet and exercise regimens, as well as multiple glucose-monitoring options.

\section{Long-Term}

- Added help within the simulation rather than Diplomates having to exit the simulation and review the tutorial to get help with particular features.

- Removed the wire cage human form in the left panel of the screen and attached all this form's physical examination findings to the appropriate buttons in the right hand frame.

- Provided immediate access in the left frame to the previous visit's abnormal findings.

- Implemented between-visit access to and ordering of laboratories and tests.
- Provided diet, exercise, referral and consultation options as menu items in the treatment frame.

- Provided the ability to advance the simulation several minutes at a time within the current visit.

- Created zooming capability for electrocardiograms and other image media.

- Created more context appropriate responses to Diplomate queries.

\section{Friction between the ABFM and AAFP}

The implementation of the MC-FP process has caused some debate between the American Board of Family Medicine and the American Academy of Family Physicians (AAFP). The ABFM and the AAFP both signed a Memorandum of Understanding in the spring of 2003 stating the 2 organizations were committed to a collaborative approach to discerning and responding to physicians' information needs about MC-FP. The ABFM has made numerous changes, as shown above, and continues to improve the MC-FP process after continual review of feedback from Diplomates. However, the AAFP Congress of Delegates, at their October 2004 meeting, passed a resolution encouraging the ABFM to suspend the SAMs as an MC-FP requirement "until technical and clinical problems are adequately resolved." Dr. David Price, a current member of the ABFM Board of Directors, attempted to address these concerns at the 2004 Congress of Delegates in Orlando. Although he acknowledged that the MC-FP is a work in progress, he cautioned delegates not, in effect, "to throw the baby out with the bath water." He noted that MC-FP has been designed to enhance patient care and clinical outcomes.

Another concern the AAFP raised was a perceived lack of communication from the ABFM to its Diplomates. Despite ongoing efforts from the $\mathrm{ABFM}$ and the AAFP, many Diplomates feel they do not have a complete understanding of the MC-FP process and its requirements. The AAFP has recommended a collaborative plan be developed between the AAFP and the ABFM to educate Diplomates on the process and importance of MC-FP.

Dr. Tom Norris, President of the ABFM, recently wrote a letter to all its Diplomates updating them on the MC-FP process and also addressing the concerns of the AAFP. He assured the Diplomates that the ABFM is increasing its communication efforts through many different avenues. 
Dr. Norris also addressed the concern that some physicians have experienced technical problems with the SAMs. The ABFM extensively tested the programs before their release, but there are always unforeseen problems. The ABFM encourages feedback from its Diplomates and continues to significantly improve the MC-FP process based on this important information, as can be observed from the many modifications that have already been made. Although the MC-FP will continue to evolve, Dr. Norris reiterated that the ABFM believes any suspension of the program, as the AAFP has suggested, would do more harm than good, stating that the program is grounded in sound educational theory and will enhance the status and image of family physicians.

\section{Diplomate Feedback}

One technical problem that the ABFM has addressed as a result of Diplomate feedback is the lack of adequate access in many areas. The ABFM solved this problem by offering a downloadable version of the questions that may be printed and completed off-line. The answers can then later be re-entered into the computer. In addition, there were a small percentage of diplomates who experienced difficulties with their hardware configurations, such as well-known, industry-wide problems with Apple computers running Internet Explorer. Although already working toward solving this issue, the ABFM added additional technical support.

A concern with security issues has also been raised by some Diplomates. Many institutions limit the types of programs that will run on their computers. The ABFM developed a Help Desk to assist Diplomates experiencing problems in this area. The Help Desk has also assisted Diplomates who have felt that the SAMs take too much time at the computer. The ABFM has additionally addressed this issue by offering a review mode after the first attempt at the questions.

Last, the ABFM has addressed the concern of lack of communication regarding the introduction of MC-FP. The ABFM developed a communications group within the ABFM to specifically handle these efforts. This development has led to more updated material on the web site, push e-mails to eligible Diplomates, and mass mailings to all Diplomates. In addition, the ABFM has distributed educational materials to AAFP State Chapters for their use in developing educational programs.

As MC-FP continues to evolve, the ABFM will continue to address any issues or problems as they arise. Although much of the publicized discussion regarding the MC-FP program in the last year has been negative, the ABFM has also received very positive feedback from a number of Diplomates after they completed their first MC-FP SAM requirement. One Diplomate said, "It was a very positive experience overall, and I would not want to go back to written format ever again." Another diplomate concurred, saying he found it to be "a valuable learning experience." He added, "The SAMs and Clinical Simulations are so good that they provide a better assessment of one's knowledge than attending multiple CME meetings and review courses."

Yet another Diplomate who recently completed the Diabetes SAM applauded the ABFM, saying "Not only have you devised an online experience that was instructive and rewarding, but the developers of the online program also should be commended for their design. I rarely have seen such programs work this well in the first year of operation." He ended saying, "I am now of the opinion that the maintenance of certification experience was exceedingly worth the time and expense. Not only was the online program designed well, but in the end, I also felt as though I had had a comprehensive review of the subject matter and that I had significantly improved my knowledge, understanding and skills regarding diabetic diagnosis and management."

The ABFM will continue to encourage feedback from its Diplomates, whether positive or negative, so that we can continue to improve the MC-FP program. The ABFM believes that the MC-FP program will play a significant role in assisting board-certified family physicians in providing even better care to their patients. As a result of this process, we will be able to assure the American public that every American Board of Family Medicine Diplomate has met the highest standards of accountability. 